\title{
Integrated analysis of miRNA and mRNA expression in the blood of patients with Alzheimer's disease
}

\author{
ZONGWEN WANG*, LEI SHEN*, YUE WANG and SHUQI HUANG \\ Neurology Department, Shanghai Tianyou Hospital, Shanghai 200040, P.R. China
}

Received October 22, 2019; Accepted April 17, 2020

DOI: $10.3892 / \mathrm{mmr} .2020 .11162$

\begin{abstract}
Alzheimer's disease (AD) is a progressive neurodegenerative disease, which is considered the most common type of dementia worldwide. The aim of the present study was to identify key microRNAs (miRNAs/miRs) and mRNAs affecting the pathogenesis of $\mathrm{AD}$, which may be developed as promising biomarkers for the early diagnosis or targeted therapy of patients with AD. Integrative analysis was performed on 12 representative miRNA datasets and three mRNA datasets of the blood from patients with AD, in order to identify differentially expressed (DE)miRNAs and DEmRNAs. Subsequently, the miRWalk database was used to identify the potential miRNA-mRNA interactions among DEmiRNAs and DEmRNAs, and an AD-specific miRNA-mRNA network was constructed using Cytoscape software. Gene Ontology and Kyoto Encyclopedia of Genes and Genomes pathway enrichment analyses were performed to assess the target mRNAs of DEmiRNAs. A total of 37 DEmiRNAs and 2,011 DEmRNAs were identified between AD and normal control samples. In addition, 853 high confidence miRNA-mRNA interactions were identified and subsequently used to construct the AD specific miRNA-mRNA network. A total of five miRNAs, including hsa-miR-93, hsa-miR-26b, hsa-miR-34a, hsa-miR-98-5p and hsa-miR-15b-5p were identified as the key nodes in the miRNA-mRNA network by topological analysis. Functional enrichment analysis demonstrated that the target mRNAs of DEmiRNAs were enriched in AD-associated pathways, such as the 'neurotrophin signaling pathway' and 'insulin signaling pathway'. Taken together, the results of the present study provide novel insights into the molecular mechanisms underlying AD and contribute to the identification of biomarkers and novel strategies for drug design for $\mathrm{AD}$ treatment.
\end{abstract}

Correspondence to: Dr Shuqi Huang, Neurology Department, Shanghai Tianyou Hospital, 528 Zhennan Road, Putuo, Shanghai 200040, P.R. China

E-mail: dochuang1127@126.com

${ }^{*}$ Contributed equally

Key words: Alzheimer's disease, miRNA profiles, mRNA profiles, miRNA-mRNA network, biomarkers

\section{Introduction}

Alzheimer's disease (AD) is the most common form of senile dementia characterized by neuronal death, loss of synaptic function and atrophy in areas of the brain that affect cognitive functions and memory $(1,2)$. The prevalence of $\mathrm{AD}$ is estimated to triple by 2050 , increasing significant economic and social burden on patients and society (3). Currently, cognitive testing and neuroimaging remain the gold standard for the diagnosis of $\mathrm{AD}$ (4); however, these clinical techniques are complicated and expensive (5). Thus, simple and convenient biomarkers are critically required to improve diagnosis of early stage AD (6). Screening for biomarkers in patients with $\mathrm{AD}$ is predominantly reported for cerebrospinal fluid, blood and other biological samples, such as urine, breath and saliva $(7,8)$. Increasing evidence has demonstrated that detection of biomarkers in peripheral blood is minimally invasive, low-cost and easily applied for mass screening $(9,10)$.

MicroRNAs (miRNAs/miRs) are 22-23 nucleotide long small non-coding RNAs, which suppress gene expression through binding to the 3 '-untranslated region of corresponding mRNAs (11). It has been reported that miRNAs are ideal biomarkers due to their stability in body fluids, and can be attributed to specific organs and pathologies in AD (12). For example, Hara et al (13) demonstrated that hsa-miR-501-3p may be a serum biomarker that could correspond to pathological events occurring in the brain of patients with AD. Additionally, Jia and Liu (14) reported that downregulated hsa-miR-223 serum may serve as a biomarker in $\mathrm{AD}$, as demonstrated by quantitative PCR analysis of serum samples from 84 probable sporadic patients with $\mathrm{AD}$ and 62 healthy individuals in China.

Currently, a number of studies have identified several miRNAs or mRNAs that are significantly differentially expressed (DE) in the blood from patients with AD compared with normal control samples, indicating their key functions in the pathogenesis of $\mathrm{AD}(13,14)$. However, the comparability of these studies is particularly challenging due to their small sample size, as well as differences in their quantification methods and protocols. There is a need to combine the study results using a meta-analysis approach to improve the understanding of the molecular mechanisms underlying AD. Chen et al (15) analyzed nine representative miRNA datasets of AD samples, which originated from tissues, serum, extracellular or cerebrospinal fluid, and identified 13 key miRNAs associated with AD. Cătană et al (16) evaluated the diagnostic 
value of miRNAs expressed in different body fluids of patients with AD using two meta-analytical approaches with different statistic indicators. However, a detailed map of specific biomarkers in the blood of patients with AD is still lacking.

The present study systematically analyzed 15 representative miRNA and mRNA datasets of the blood from patients with AD using a series of bioinformatics methods. The present study identified several key miRNAs, mRNAs and pathways affecting the pathogenesis of $\mathrm{AD}$, providing novel insights into the molecular mechanisms underlying AD.

\section{Materials and methods}

miRNA and mRNA expression datasets. The miRNA and mRNA expression profiles of patients with AD were downloaded from the Gene Expression Omnibus (GEO) database (http://www.ncbi.nlm.nih.gov/geo), which is a public repository for high-throughput gene expression datasets (17). The present study collected 12 representative miRNA and three mRNA expression profiles of blood samples from patients with AD and normal control samples. All datasets contained at least three $\mathrm{AD}$ samples and had age-matched normal control samples in each group. Among the 12 miRNA expression datasets, seven focused on serum $(9,10,13,14,18-20)$, three on plasma (21-23), one on both serum and plasma (24), and one focused on whole blood (25). The three mRNA expression datasets all focused on whole blood (5). Detailed information of these datasets are presented in Table I.

Data processing and differential expression analysis. The DEmiRNA information was manually extracted from the publications of 12 miRNA datasets. Only the miRNAs validated by previous reverse transcription-quantitative PCR analysis were retained and categorized into upregulated and downregulated miRNAs in patients with AD compared with normal control samples (Table I). The miRNAs identified in at least one dataset were integrated as high-confidence DEmiRNAs.

The raw data of three mRNA expression profiles (GSE63060, GSE63061 and GSE18309) were downloaded from the GEO database and preprocessed with background correction. Subsequently, the Limma package in $\mathrm{R}$ language (version 3.40.6; https://bioconductor.org/packages/limma) was used to normalize the datasets and identify the significantly DEmRNAs with the following cut-off criteria: Adjusted $\mathrm{P}<0.05$ and $\log$ fold change $(\log \mathrm{FC}) \mid>0.5$. In the case where multiple probes corresponded to the same gene, the probe with the maximal value was selected as the expression of that particular gene. The DEmRNAs were clustered using hierarchical clustering and implemented by pheatmap package in $\mathrm{R}$ language (version 1.0.12) (26). Euclidean distance was selected as a measure of distance between the samples.

Prediction of miRNA-mRNA interactions. The putative target mRNAs of high-confidence DEmiRNAs were predicted using six bioinformatic algorithms [DIANA-microT (27), miRanda (28), miRDB (29), miRWalk (30), PICTAR (31) and TargetScan (32)]; the default parameters were used for all software programs, and target mRNAs identified by at least four algorithms were retained. Subsequently, target
mRNAs identified in the miRWalk database (http://www. umm.uni-heidelberg.de/apps/zmf/mirwalk) were selected, which collects data on experiment supported miRNA-mRNA interactions (30). Considering miRNAs suppress expression of their target mRNAs, the DEmRNAs whose expression were inversely associated with the miRNAs were regarded as the miRNA target.

Construction of miRNA-mRNA network and identification of hub nodes. The high-confidence DEmiRNA-mRNA interactions were used to construct the miRNA-mRNA network using Cytoscape software (version 3.5.0; http://www.cytoscape.org). The hub nodes in the network were nodes with high scores of network topology property indictors, which were analyzed using CytoNCA (version 2.1.6) within Cytoscape, including degree centrality, betweenness centrality and closeness centrality. In general, a high score of network topology property indictors indicates important roles in the network.

Functional annotation. Gene Ontology (GO) analysis, which organizes genes into hierarchical categories and determines the gene regulatory network on the basis of biological process (BP), molecular function (MF) and cellular component (CC), was applied to analyze the functions of genes. Kyoto Encyclopedia of Genes and Genomes (KEGG) pathway analysis was used to determine which signaling pathways the genes were enriched in. The Database for Annotation, Visualization and Integrated Discovery (DAVID; https:/david.ncifcrf.gov) was used for both GO and KEGG enrichment analyses where a false discovery rate (FDR) $<0.05$ was considered to indicate a statistically significant difference (33).

\section{Results}

Differential analysis of miRNAs and mRNAs in patients with $A D$. The workflow of the present study is presented in Fig. 1 . The present study downloaded 12 separate miRNA expression profiling datasets of blood samples from patients with $\mathrm{AD}$ and normal control samples. Detailed information on sample size, blood component and experimentally supported DEmiRNAs were manually extracted from the references of the datasets (Table I). miRNAs identified in at least one dataset were integrated as high-confidence DEmiRNAs. A total of 37 miRNAs were identified to be significant DEmiRNAs, among which seven miRNAs were upregulated and 30 miRNAs were downregulated in patients with $\mathrm{AD}$ compared with normal control samples.

A total of three mRNA expression profiles of whole blood samples from patients with AD and normal control samples were downloaded from the publicly accessible database GEO (Table I). Following background correction and normalization, 2,011 DEmRNAs were identified using the Limma package, under the following cut-off criteria; adjusted $\mathrm{P}<0.05$ and $\mid \log \mathrm{FCl}>0.5$. Among these, $911 \mathrm{mRNAs}$ were upregulated and 1,100 were downregulated in patients with AD compared with normal control samples. Subsequently, hierarchical clustering of the DEmRNAs was performed, which is displayed in the heatmap (Fig. 2).

Construction of the miRNA-mRNA network and identification of hub nodes. The DEmiRNAs-mRNA interactions 


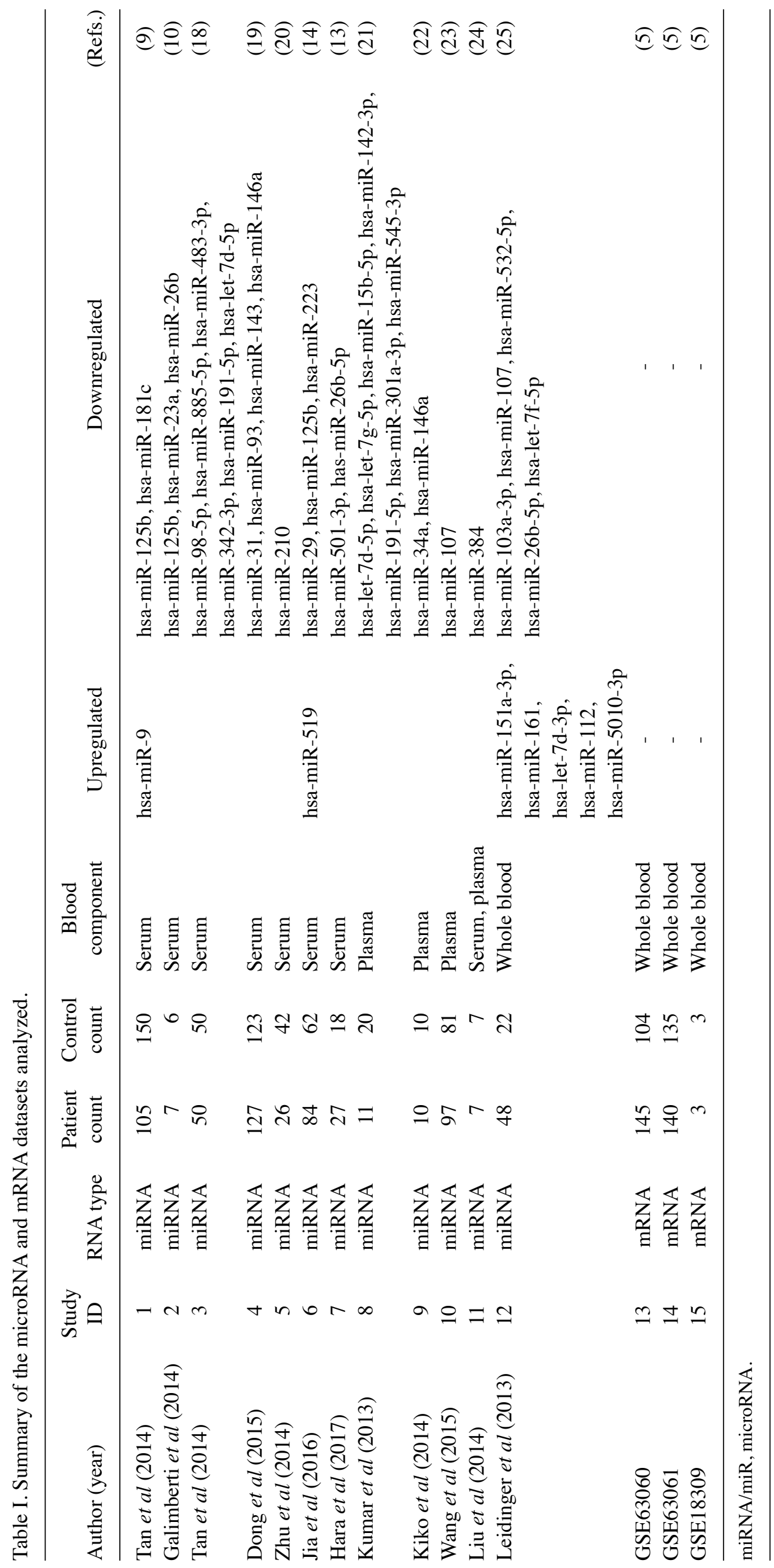




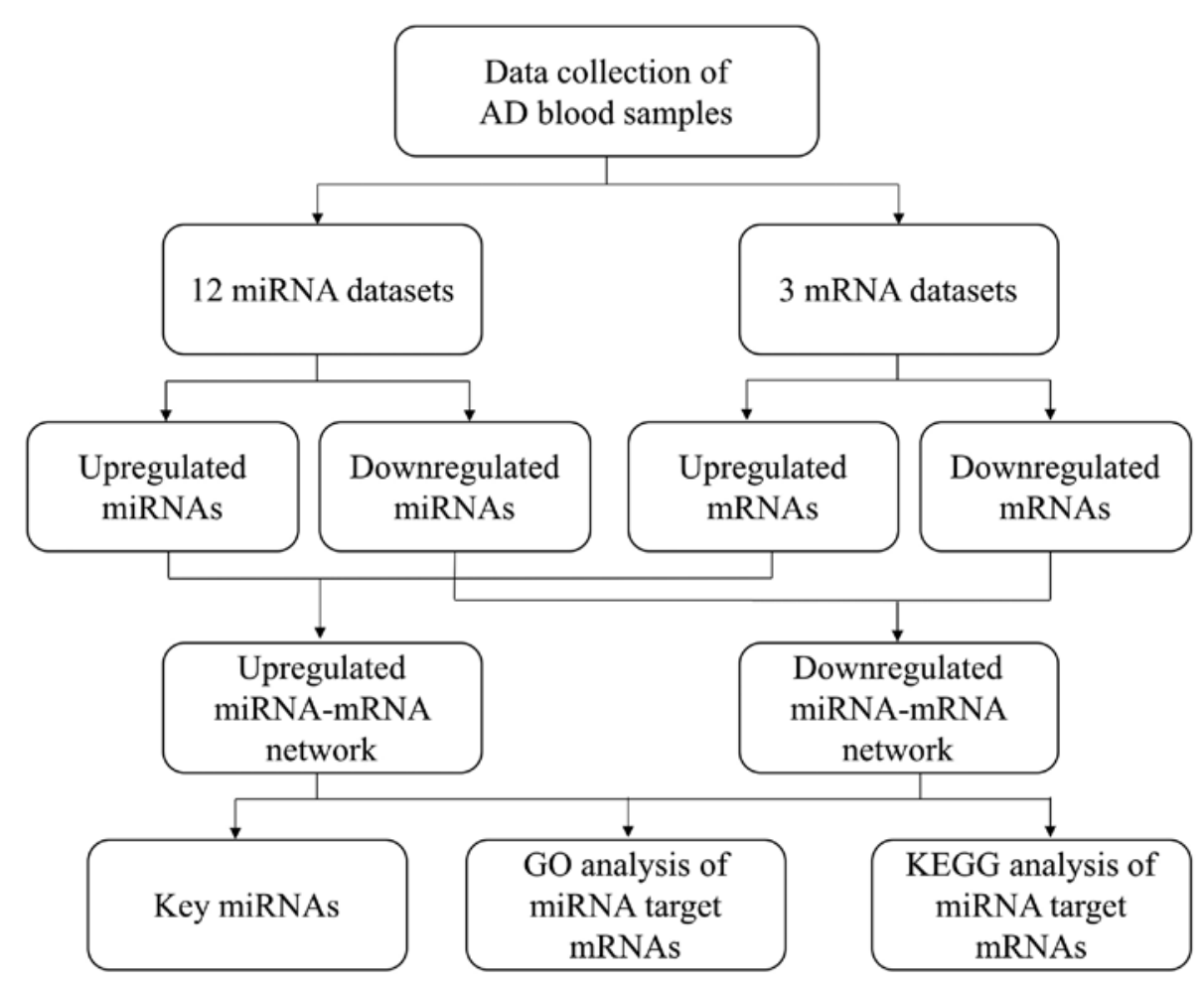

Figure 1. Schematic diagram of the study workflow to determine the association between microRNA and AD. AD, Alzheimer's disease; miRNA, microRNA; GO, Gene Ontology; KEGG, Kyoto of Encyclopedia of Genes and Genomes.

were identified using four algorithms and validated by subsequent experimentation in the miRWalk database. Target mRNAs of the DEmiRNAs were inversely associated with the expression of corresponding miRNAs between patients with AD and normal control samples. In total, 853 high-confidence DEmiRNA-mRNA interactions were identified, of which 17 were upregulated miRNAs-mRNA and 836 were downregulated miRNAs-mRNA interactions. The upregulated miRNAs-mRNA network and downregulated miRNAs-mRNA network were constructed based on these miRNA-mRNA interactions using Cytoscape software. The upregulated miRNAs-mRNA network consisted of 20 nodes and 17 edges (Fig. 3A). Of these, hsa-miR-9 had the highest connectivity and was demonstrated to negatively interact with 14 target mRNAs. A high score of network topology property indictors suggests a notable role in the network. According to the rankings of network topology property indictors, including degree centrality, betweenness centrality and closeness centrality, the top five nodes of the downregulated miRNAs-mRNA network, which consisted of 413 nodes and 836 edges, are listed in Table II. A total of five miRNAs, including hsa-miR-93, hsa-miR-26b, hsa-miR-34a, hsa-miR-98-5p and hsa-miR-15b-5p were among the top nodes for all topology property indictors, suggesting their critical roles in the pathogenesis of AD. The interactions of these five key miRNAs and their target mRNAs are presented in Fig. 3B.

GO terms annotation of the target mRNAs of DEmiRNAs. The online tool DAVID was used to identify significantly enriched GO terms for the target mRNAs of DEmiRNAs between patients with AD and normal control samples (Table III). The results indicated that the target mRNAs were predominantly enriched in BP terms, including 'regulation of transcription' and 'apoptotic process'. Regarding CC, the target mRNAs were enriched in the 'nucleus' and 'intracellular'. In addition, MF analysis displayed that the target mRNAs were significantly enriched in 'metal ion binding' and 'DNA binding'.

KEGG pathway enrichment of the target mRNAs of DEmiRNAs. The significantly enriched pathways of the target mRNAs of DEmiRNAs between patients with AD and normal control samples are presented in Table IV. The results demonstrated that the target mRNAs were enriched in the 'neurotrophin signaling pathway', 'insulin signaling pathway', 'MAPK signaling pathway', 'lysosome', 'Alzheimer's disease' and 'Huntington's disease' (Fig. 4).

\section{Discussion}

Increasing evidence has demonstrated that identifying biomarkers using meta-analysis is helpful to the diagnosis and targeted therapy of patients with AD at an early stage $(15,16)$. However, previous studies have used datasets that originate from different samples, for example, Chen et al (15) combined datasets of tissues, serum, extracellular and cerebrospinal fluid, and Cătană et al (16) evaluated datasets from different body fluids. Conversely, the present study focused on datasets from the blood. In addition, the strategies of these previous studies only considered miRNAs, whereas the present study identified key miRNAs, mRNAs and pathways affecting the pathogenesis of AD by integrating the miRNA and mRNA expression profiling datasets. Although different strategies were used, some of the key miRNAs identified in the present study were in agreement with previous studies, for example, hsa-miR-26b, hsa-miR-15b 


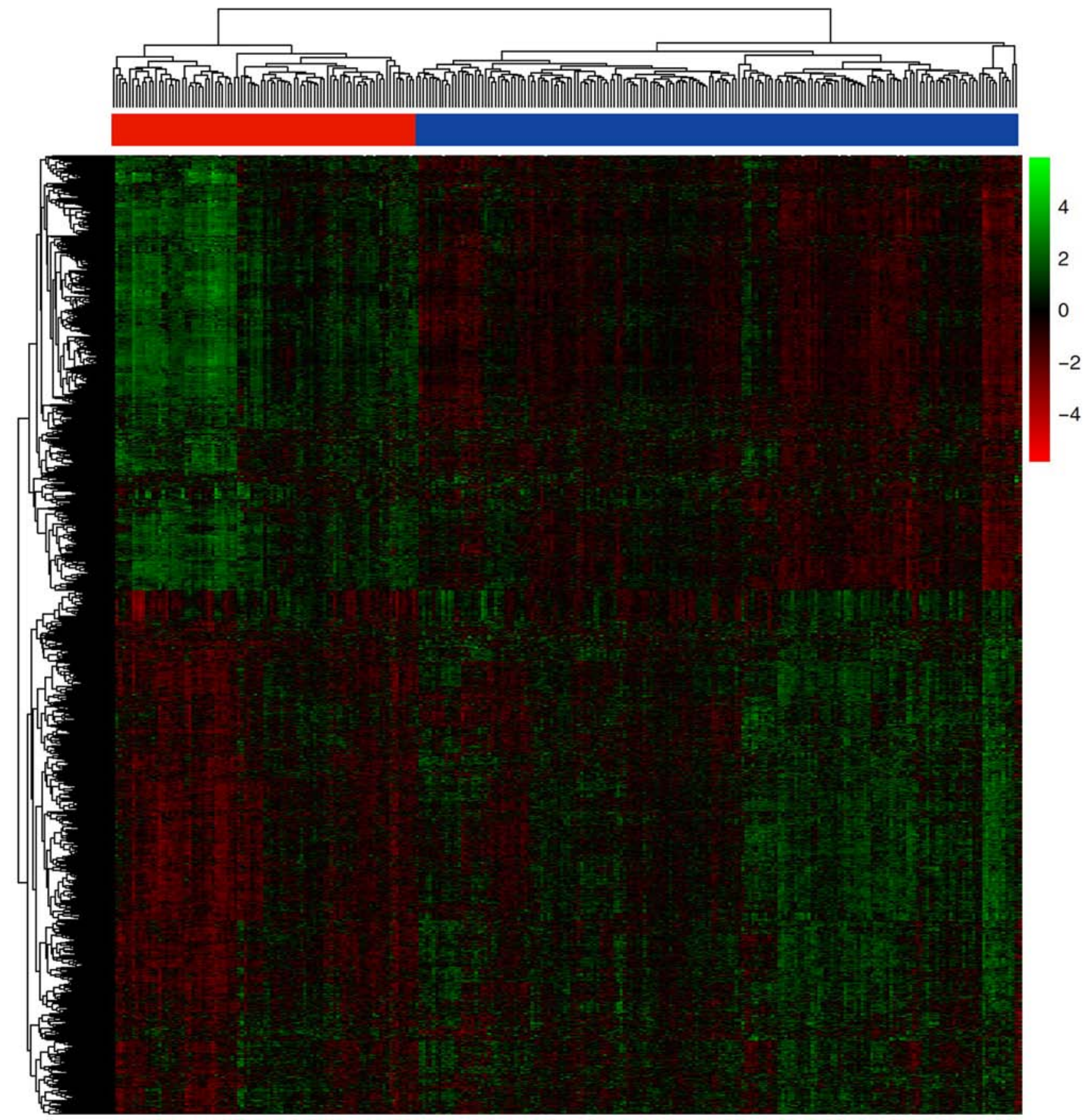

Figure 2. Heatmap of cluster analysis of differentially expressed mRNAs between patients with AD and normal control samples. Rows represent genes and columns represent samples. The values indicate the expression levels of the genes. Red represents normal control blood samples and blue represents blood samples from patients with AD. AD, Alzheimer's disease.

and hsa-miR-93 were also identified by Chen et al (15). In the present study, the DEmiRNA-mRNA crosstalk was assessed between the blood from patients with AD and normal control samples, by integrating the largest count of miRNA and mRNA datasets. Topological analysis of the AD-specific miRNA-mRNA network identified five miRNAs, including hsa-miR-93, hsa-miR-26b, hsa-miR-34a, hsa-miR-98-5p and hsa-miR-15b-5p as hub nodes, suggesting their critical roles in the pathogenesis of AD. Functional enrichment analysis demonstrated that the target mRNAs of DEmiRNAs were enriched in AD-associated pathways, such as the "neurotrophin signaling pathway' and 'insulin signaling pathway'.
hsa-miR-93 was the hub node with the highest topology property indictors, which was demonstrated to negatively regulate 87 DEmRNAs in the AD-specific downregulated miRNA-mRNA network, including GADPH, ATP synthase F1 subunit $\beta$ (ATP5B) and MAPK1. These protein-coding genes were significantly upregulated in patients with AD and enriched in the AD-associated pathway in the present study. Dong et al (19) reported that hsa-miR-93 was markedly decreased in the serum of patients with AD compared with controls, while screening the expression profile of serum miRNAs by Solexa sequencing. In addition, it was demonstrated that the panel of hsa-miR-93, along with hsa-miR-31 

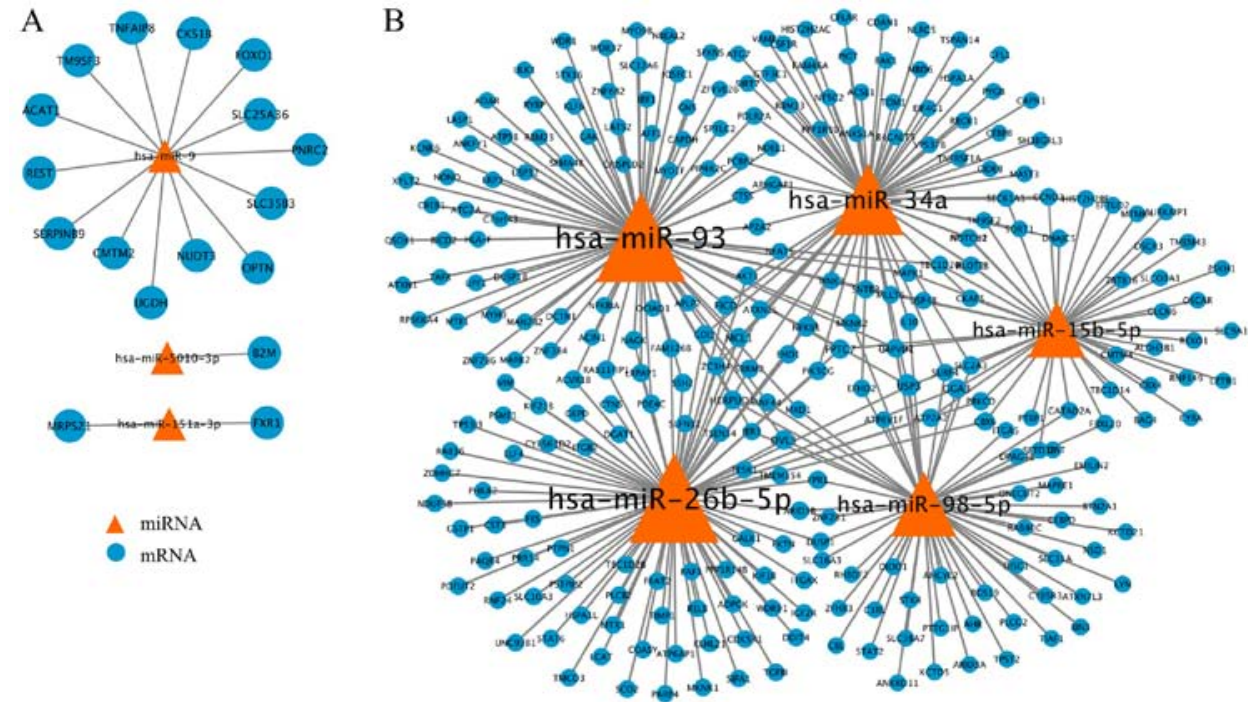

Figure 3. DEmiRNAs-mRNA regulatory network in Alzheimer's disease. (A) Upregulated and (B) downregulated miRNA-mRNA network of the key five miRNAs. Orange triangles represent miRNAs and blue circles represent target mRNAs. The solid lines represent high-confidence DEmiRNA-mRNA interactions. DE, differentially expressed; miRNA, microRNA.

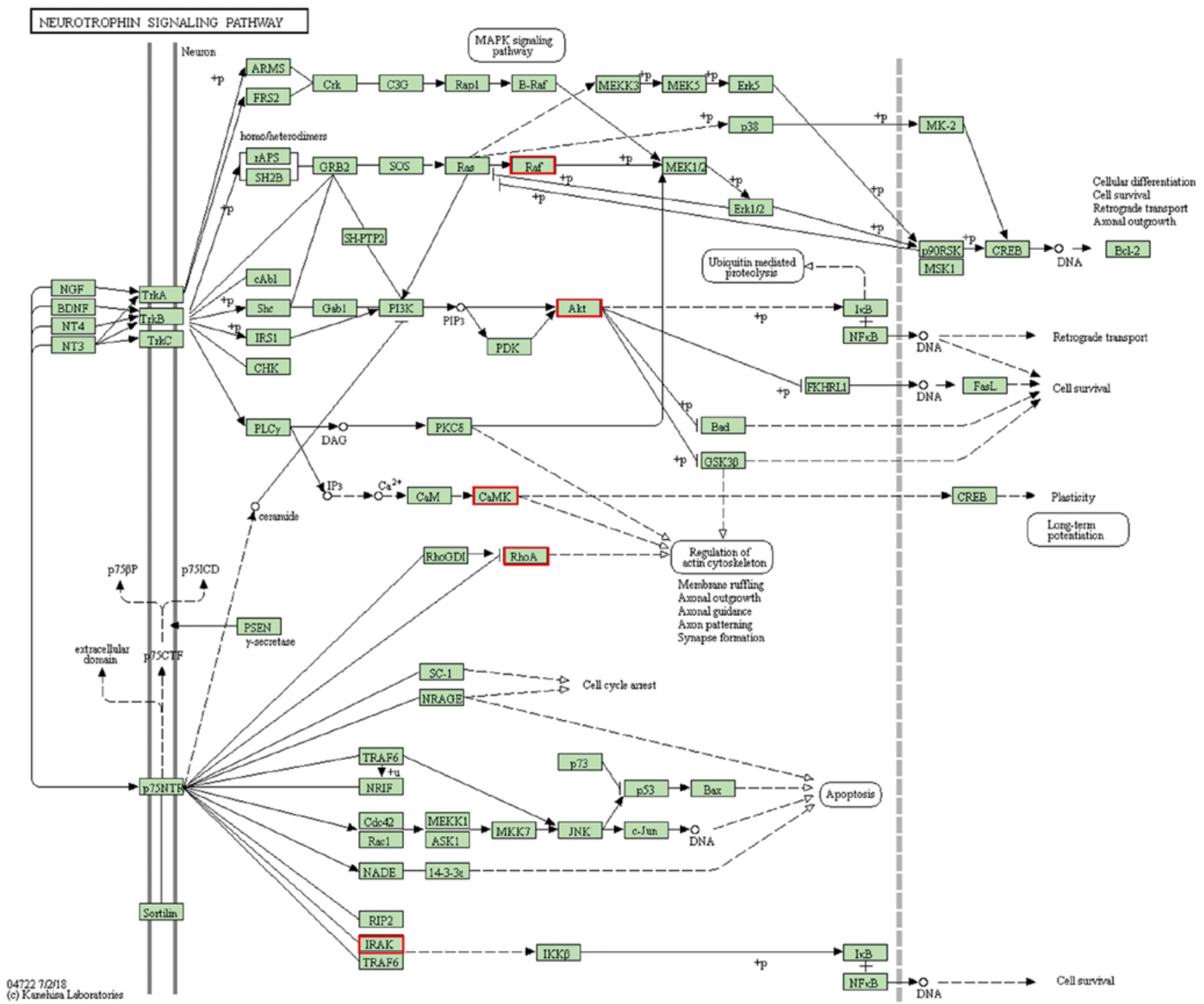

Figure 4. Neurotrophin signaling pathway is enriched in target mRNAs of differentially expressed microRNAs in Alzheimer's disease. Red rectangles represent the target mRNAs that are enriched in the neurotrophin signaling pathway. The image was obtained from the Kyoto Encyclopedia of Genes and Genomes database (https://www.kegg.jp/kegg-bin/show_pathway?map=hsa04722\&show_description=show). 
Table II. Top five nodes in the downregulated miRNA-mRNA network according to the score of network topology property indictors.

\begin{tabular}{llccc}
\hline Rank & \multicolumn{1}{c}{ Node } & Degree & Betweenness & Closeness \\
\hline 1 & hsa-miR-93 & 87 & 197.28 & $46,730.89$ \\
2 & hsa-miR-26b & 87 & 194.30 & $25,361.43$ \\
3 & hsa-miR-34a & 66 & 180.78 & $32,132.43$ \\
4 & hsa-miR-98-5p & 58 & 173.52 & $21,099.35$ \\
5 & hsa-miR-15b-5p & 48 & 170.25 & $20,266.93$ \\
\hline
\end{tabular}

Table III. Significantly enriched Gene Ontology terms of the target mRNAs of differentially expressed microRNAs identified by The Database for Annotation, Visualization and Integrated Discovery.

\begin{tabular}{|c|c|c|}
\hline GO ID & GO terms & FDR \\
\hline \multicolumn{3}{|c|}{ Biological process } \\
\hline GO:0006355 & Regulation of transcription, DNA-dependent & $4.41 \times 10^{-5}$ \\
\hline GO:0006915 & Apoptotic process & $6.38 \times 10^{-5}$ \\
\hline GO:0006954 & Inflammatory response & $7.75 \times 10^{-3}$ \\
\hline GO:0007165 & Signal transduction & $2.34 \times 10^{-5}$ \\
\hline GO:0006468 & Protein phosphorylation & $4.63 \times 10^{-8}$ \\
\hline GO:0008360 & Regulation of cell shape & $4.84 \times 10^{-2}$ \\
\hline GO:0008283 & Cell proliferation & $9.85 \times 10^{-4}$ \\
\hline GO:0044419 & Interspecies interaction between organisms & $1.64 \times 10^{-7}$ \\
\hline GO:0006810 & Transport & $1.62 \times 10^{-2}$ \\
\hline GO:0045944 & Positive regulation of transcription from RNA polymerase II promoter & $5.45 \times 10^{-6}$ \\
\hline \multicolumn{3}{|c|}{ Molecular function } \\
\hline GO:0046872 & Metal ion binding & $1.12 \times 10^{-9}$ \\
\hline GO:0003677 & DNA binding & $1.53 \times 10^{-4}$ \\
\hline GO:0008270 & Zinc ion binding & $4.04 \times 10^{-4}$ \\
\hline GO:0003700 & Sequence-specific DNA binding transcription factor activity & $4.85 \times 10^{-4}$ \\
\hline GO:0016740 & Transferase activity & $3.39 \times 10^{-2}$ \\
\hline GO:0005524 & ATP binding & $1.81 \times 10^{-12}$ \\
\hline GO:0005515 & Protein binding & $1.42 \times 10^{-35}$ \\
\hline GO:0000166 & Nucleotide binding & $1.12 \times 10^{-15}$ \\
\hline GO:0043565 & Sequence-specific DNA binding & $2.33 \times 10^{-3}$ \\
\hline GO:0016301 & Kinase activity & $6.54 \times 10^{-4}$ \\
\hline \multicolumn{3}{|c|}{ Cellular component } \\
\hline GO:0005634 & Nucleus & $1.54 \times 10^{-21}$ \\
\hline GO:0005622 & Intracellular & $1.40 \times 10^{-6}$ \\
\hline GO:0016607 & Nuclear speck & $2.21 \times 10^{-3}$ \\
\hline GO:0016021 & Integral to membrane & $8.15 \times 10^{-5}$ \\
\hline GO:0005625 & Soluble fraction & $6.85 \times 10^{-3}$ \\
\hline GO:0005794 & Golgi apparatus & $7.52 \times 10^{-5}$ \\
\hline GO:0016020 & Membrane & $7.88 \times 10^{-16}$ \\
\hline GO:0005783 & Endoplasmic reticulum & $3.40 \times 10^{-5}$ \\
\hline GO:0005737 & Cytoplasm & $5.10 \times 10^{-26}$ \\
\hline GO:0005739 & Mitochondrion & $7.82 \times 10^{-5}$ \\
\hline
\end{tabular}

GO, Gene Ontology; FDR, false discovery rate.

and miR-146a, may be used to discriminate AD from vascular dementia. Takashima et al (34) reported that hsa-miR-93 may be a potential prognostic biomarker in primary central nervous system lymphoma. GAPDH is a family of abundantly 


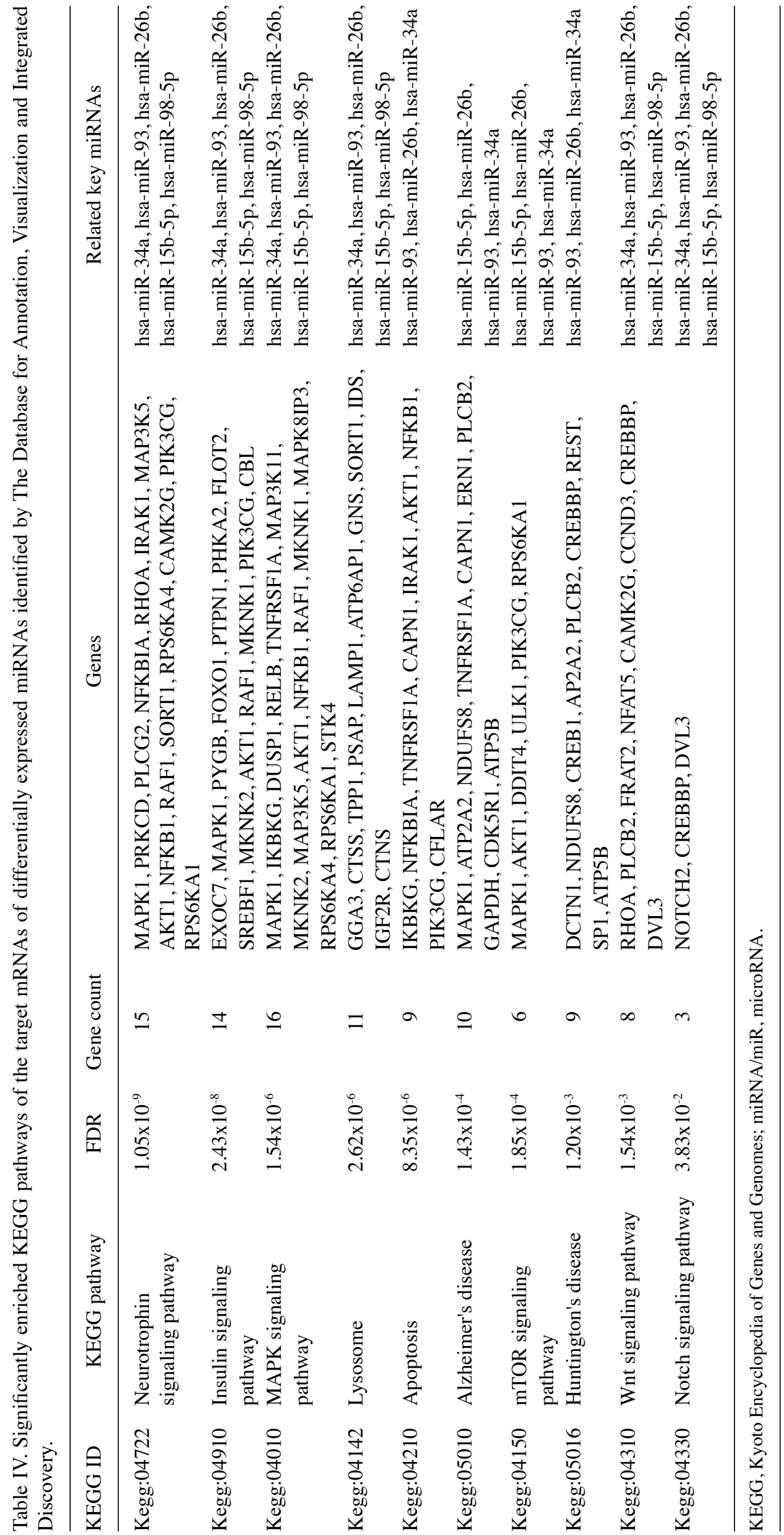


expressed oxidoreductases that are known for their role in glucose metabolism (35). It has been reported that GAPDH is able to interact with various small molecules (proteins and membranes) that serve key roles in normal and pathological cell functions, including AD-associated proteins and the $\beta$-amyloid precursor protein (36). It was therefore hypothesized that decreased hsa-miR-93 expression may serve vital roles in the pathological process of AD by targeting GADPH, ATP5B and MAPK1.

hsa-miR-26b was another hub node identified in the present study that negatively regulated 80 DEmRNAs in the AD downregulated miRNA-mRNA network, including cyclin-dependent kinase 5 regulatory subunit 1 (CDK5R1), ATPase sarcoplasmic/endoplasmic reticulum $\mathrm{Ca}^{2+}$ transporting 2, AKT1 and NF-kB1, which were enriched in the AD pathway and 'neurotrophin signaling pathway'. Consistent with previous findings, the results of the present study demonstrated that hsa-miR-26b was significantly downregulated in patients with AD compared with the normal control samples, whereas the corresponding mRNAs were upregulated in patients with AD (25,37). CDK5R1 encodes for p35, a protein required for the main activation of CDK5 (38). The active p35/CDK5 complex has been reported to be involved in several aspects of brain development and function, and its deregulation is closely associated with AD onset and progression (39). Taken together, the results of the present study suggested that hsa-miR-26b may negatively regulate CDK5R1 expression in AD.

The 'neurotrophin signaling pathway' and 'insulin signaling pathway' were the most significantly enriched pathways of the target mRNAs of DEmiRNAs between patients with $\mathrm{AD}$ and normal control samples. The neurotrophin signaling pathway is activated by neurotrophins through binding to the tyrosine protein kinase receptor family, which results in a series of neuronal functions, such as axonal growth, cell survival, cell differentiation, dendritic arborization, synapse formation, plasticity and axonal guidance $(40,41)$. The insulin signaling pathway, which is the main signal transduction pathway in insulin physiological function, serves a vital role in the metabolism, nerve protection and regulation of cognitive dysfunction (42). Increasing evidence has demonstrated that the symptoms of patients with AD are consistently accompanied with a disordered insulin signaling pathway or other symptoms, suggesting that the insulin signaling pathway may be closely associated with the pathogenesis of AD (43).

In conclusion, availably representative miRNA and mRNA expression profiling datasets of the blood from patients with AD were collected and subjected to comprehensive analysis though a series of bioinformatics methods in the present study. The AD-specific miRNA-mRNA crosstalk network was constructed and several key dysregulated miRNAs, mRNAs and signaling pathways affecting the pathogenesis of AD were identified. However, further experimental studies testing these results would be desirable. Taken together, the results of the present study provided a valuable resource for depicting the complexity of $\mathrm{AD}$, and may contribute to the development of diagnostic biomarkers and therapeutic targets for AD.

\section{Acknowledgements}

Not applicable.

\section{Funding}

No funding was received.

\section{Availability of data and materials}

The datasets used and/or analyzed during the present study are available from the corresponding author on reasonable request.

\section{Authors' contributions}

SH and ZW conceived and designed the study; SH, ZW and LS analyzed and interpreted the data; YW acquired the data and wrote the manuscript; and $\mathrm{SH}, \mathrm{ZW}$ and LS reviewed and edited the manuscript. All authors read and approved the final manuscript.

\section{Ethics approval and consent to participate}

Not applicable.

\section{Patient consent for publication}

Not applicable.

\section{Competing interests}

The authors declare that they have no competing interests.

\section{References}

1. Patel H, Dobson RJB and Newhouse SJ: A Meta-analysis of Alzheimer's disease brain transcriptomic data. J Alzheimers Dis 68: 1635-1656, 2019.

2. Takousis P, Sadlon A, Schulz J, Wohlers I, Dobricic V, Middleton L, Lill CM, Perneczky R and Bertram L: Differential expression of microRNAs in Alzheimer's disease brain, blood, and cerebrospinal fluid. Alzheimers Dement 15: 1468-1477, 2019.

3. Readhead B, Haure-Mirande JV, Funk CC, Richards MA, Shannon P, Haroutunian V, Sano M, Liang WS, Beckmann ND, Price ND, et al: Multiscale analysis of independent Alzheimer's cohorts finds disruption of molecular, genetic, and clinical networks by human Herpesvirus. Neuron 99: 64-82.e7, 2018.

4. Swarbrick S, Wragg N, Ghosh S and Stolzing A: Systematic review of miRNA as biomarkers in Alzheimer's disease. Mol Neurobiol 56: 6156-6167, 2019.

5. Sood S, Gallagher IJ, Lunnon K, Rullman E, Keohane A, Crossland $\mathrm{H}$, Phillips BE, Cederholm $\mathrm{T}$, Jensen $\mathrm{T}$, van Loon LJ, et al: A novel multi-tissue RNA diagnostic of healthy ageing relates to cognitive health status. Genome Biol 16: 185,2015 .

6. Liang WS, Reiman EM, Valla J, Dunckley T, Beach TG, Grover A, Niedzielko TL, Schneider LE, Mastroeni D, Caselli R, et al: Alzheimer's disease is associated with reduced expression of energy metabolism genes in posterior cingulate neurons. Proc Natl Acad Sci USA 105: 4441-4446, 2008.

7. Cheng L, Doecke JD, Sharples RA, Villemagne VL, Fowler CJ, Rembach A, Martins RN, Rowe CC, Macaulay SL, Masters CL, et al: Prognostic serum miRNA biomarkers associated with Alzheimer's disease shows concordance with neuropsychological and neuroimaging assessment. Mol Psychiatry 20: 1188-1196, 2015.

8. McKeever PM, Schneider R, Taghdiri F, Weichert A, Multani N Brown RA, Boxer AL, Karydas A, Miller B, Robertson J and Tartaglia MC: MicroRNA expression levels are altered in the cerebrospinal fluid of patients with young-onset Alzheimer's disease. Mol Neurobiol 55: 8826-8841, 2018. 
9. Tan L, Yu JT, Liu QY, Tan MS, Zhang W, Hu N, Wang YL, Sun L, Jiang T and Tan L: Circulating miR-125b as a biomarker of Alzheimer's disease. J Neurol Sci 336: 52-56, 2014.

10. Galimberti D, Villa C, Fenoglio C, Serpente M, Ghezzi L, Cioffi SM, Arighi A, Fumagalli G and Scarpini E: Circulating miRNAs as potential biomarkers in Alzheimer's disease. J Alzheimers Dis 42: 1261-1267, 2014.

11. Fei Y, Guo P, Wang F, Li H, Lei Y, Li W, Xun X and Lu F: Identification of miRNA-mRNA crosstalk in laryngeal squamous cell carcinoma. Mol Med Rep 16: 4179-4186, 2017.

12. Lau P, Bossers K, Janky R, Salta E, Frigerio CS, Barbash S, Rothman R, Sierksma AS, Thathiah A, Greenberg D, et al: Alteration of the micro RNA network during the progression of Alzheimer's disease. EMBO Mol Med 5: 1613-1634, 2013.

13. Hara N, Kikuchi M, Miyashita A, Hatsuta H, Saito Y, Kasuga K, Murayama S, Ikeuchi T and Kuwano R: Serum microRNA miR-501-3p as a potential biomarker related to the progression of Alzheimer's disease. Acta Neuropathol Commun 5: 10, 2017.

14. Jia LH and Liu YN: Downregulated serum miR-223 servers as biomarker in Alzheimer's disease. Cell Biochem Funct 34 233-237, 2016.

15. Chen J, Qi Y, Liu CF, Lu JM, Shi J and Shi Y: MicroRNA expression data analysis to identify key miRNAs associated with Alzheimer's disease. J Gene Med 20: e3014, 2018.

16. Cătană CS, Crişan CA, Opre D and Berindan-Neagoe I: Diagnostic and prognostic value of microRNAs for Alzheimer's disease: A comprehensive meta-analysis. Med Pharm Rep 93: 53-61, 2020.

17. Barrett T, Suzek TO, Troup DB, Wilhite SE, Ngau WC, Ledoux P, Rudnev D, Lash AE, Fujibuchi W and Edgar R: NCBI GEO: Mining millions of expression profiles-database and tools. Nucl Acids Res 33 (Database Issue): D562-D566, 2005.

18. Tan L, Yu JT, Tan MS, Liu QY, Wang HF, Zhang W, Jiang T and Tan L: Genome-Wide Serum microRNA expression profiling identifies serum biomarkers for Alzheimer's disease. J Alzheimers Dis 40: 1017-1027, 2014.

19. Dong H, Li J, Huang L, Chen X, Li D, Wang T, Hu C, Xu J, Zhang C, Zen K, et al: Serum MicroRNA profiles serve as novel biomarkers for the diagnosis of Alzheimer's disease. Dis Markers 2015: 625659, 2015.

20. Zhu Y, Li C, Sun A, Wang Y and Zhou S: Quantification of microRNA-210 in the cerebrospinal fluid and serum: Implications for Alzheimer's disease. Exp Ther Med 9: 1013-1017, 2015.

21. Kumar P, Dezso Z, MacKenzie C, Oestreicher J, Agoulnik S, Byrne M, Bernier F, Yanagimachi M, Aoshima K and Oda Y: Circulating miRNA Biomarkers for Alzheimer's disease. PLoS One 8: e69807, 2013.

22. Kiko T, Nakagawa K, Tsuduki T, Furukawa K, Arai H and Miyazawa T: MicroRNAs in plasma and cerebrospinal fluid as potential markers for Alzheimer's disease. J Alzheimers Dis 39: 253-259, 2014.

23. Wang T, Chen K, Li H, Dong S, Su N, Liu Y, Cheng Y, Dai J, Yang $C$ and Xiao S: The feasibility of utilizing plasma MiRNA107 and BACE1 messenger RNA gene expression for clinical diagnosis of amnestic mild cognitive impairment. J Clin Psychiatry 76: 135-141, 2015.

24. Liu CG, Wang JL, Li L and Wang PC: MicroRNA-384 regulates both amyloid precursor protein and $\beta$-secretase expression and is a potential biomarker for Alzheimer's disease. Int J Mol Med 34: $160-166,2014$

25. Leidinger P, Backes C, Deutscher S, Schmitt K, Mueller SC, Frese K, Haas J, Ruprecht K, Paul F, Stähler C, et al: A blood based 12-miRNA signature of Alzheimer disease patients. Genome Biol 14: R78, 2013.
26. Kimes PK, Liu Y, Neil Hayes D and Marron JS: Statistical significance for hierarchical clustering. Biometrics 73: 811-821, 2017.

27. Paraskevopoulou MD, Georgakilas G, Kostoulas N, Vlachos IS Vergoulis T, Reczko M, Filippidis C, Dalamagas T and Hatzigeorgiou AG: DIANA-microT web server v5.0: Service integration into miRNA functional analysis workflows. Nucleic Acids Res 41: W169-W173, 2013.

28. Enright AJ, John B, Gaul U, Tuschl T, Sander C and Marks DS: MicroRNA targets in Drosophila. Genome Biol 5: R1, 2003.

29. Liu W and Wang X: Prediction of functional microRNA targets by integrative modeling of microRNA binding and target expression data. Genome Biol 20: 18, 2019.

30. Dweep H, Sticht C, Pandey P and Gretz N: miRWalk-database: Prediction of possible miRNA binding sites by 'walking' the genes of three genomes. J Biomed Inform 44: 839-847, 2011.

31. Krek A, Grün D, Poy MN, Wolf R, Rosenberg L, Epstein EJ, MacMenamin P, da Piedade I, Gunsalus KC, Stoffel M and Rajewsky N: Combinatorial microRNA target predictions. Nat Genet 37: 495-500, 2005.

32. Agarwal V, Bell GW, Nam J and Bartel DP: Predicting effective microRNA target sites in mammalian mRNAs. Elife: 4: e05005, 2015.

33. Huang DW, Sherman BT and Lempicki RA: Systematic and integrative analysis of large gene lists using DAVID bioinformatics resources. Nat Protoc 4: 44-57, 2009.

34. Takashima Y, Kawaguchi A, Iwadate Y, Hondoh H, Fukai J, Kajiwara K, Hayano A and Yamanaka R: MicroRNA signature constituted of miR-30d, miR-93, and miR-181b is a promising prognostic marker in primary central nervous system lymphoma. PLoS One 14: e0210400, 2019.

35. Allen M, Cox C, Belbin O, Ma L, Bisceglio GD, Wilcox SL, Howell CC, Hunter TA, Culley O, Walker LP, et al: Association and heterogeneity at the GAPDH locus in Alzheimer's disease. Neurobiol Aging 33: 203.e25-e33, 2012.

36. Butterfield DA, Hardas SS and Lange MLB: Oxidatively modified Glyceraldehyde-3-phosphate dehydrogenase (GAPDH) and Alzheimer's disease: Many pathways to neurodegeneration. J Alzheimers Dis 20: 369-393, 2010

37. Chang WS, Wang YH, Zhu XT and Wu CJ: Genome-wide profiling of miRNA and mRNA expression in Alzheimer's disease. Med Sci Monit 23: 2721-2731, 2017.

38. Mateo I, Vázquez-Higuera JL, Sánchez-Juan P, RodríguezRodríguez E, Infante J, García-Gorostiaga I, Berciano J and Combarros O: Epistasis between tau phosphorylation regulating genes (CDK5R1 and GSK-3beta) and Alzheimer's disease risk. Acta Neurol Scand 120: 130-133, 2009.

39. Spreafico M, Grillo B, Rusconi F, Battaglioli E and Venturin M: Multiple layers of CDK5R1 regulation in Alzheimer's disease implicate long non-coding RNAs. Int J Mol Sci 19: pii: E2022, 2018.

40. Puglielli L: Aging of the brain, neurotrophin signaling, and Alzheimer's disease: Is IGF1-R the common culprit? Neurobiol Aging 29: 795-811, 2008.

41. Karki R, Kodamullil AT and Hofmann-Apitius M: Comorbidity Analysis between Alzheimer's disease and type 2 diabetes mellitus (T2DM) based on shared pathways and the role of T2DM drugs. J Alzheimers Dis 60: 721-731, 2017.

42. Jasmin B: Impairment of insulin signalling pathway in Alzheimer's disease. University of Wuzburg, Faculty of Science, $1-13,2012$.

43. Liu Y, Liu F, Grundke-Iqbal I, Iqbal K and Gong CX: Deficient brain insulin signalling pathway in Alzheimer's disease and diabetes. J Pathol 225: 54-62, 2011. 